\title{
The “Great Imitator” Presents with Abnormal LIVER ENZYMES
}

\author{
Ikumi Suzuki, MS IV, Nicholas Orfanidis, MD, Stephanie Moleski, MD, Leo C Katz, MD, David Kastenberg, MD
}

\section{Case Report}

A 28 year-old male was referred to the gastroenterology clinic for evaluation of abnormal liver enzymes. Three months prior to presentation he was evaluated at a local emergency department for vomiting and upper abdominal pain. He described pain as epigastric and worse after eating. He denied fever, chills or changes in his urine. At that time, the patient was not jaundiced but reported having a rash. Laboratory testing was remarkable for elevated liver enzymes (Table 1). The patient was advised to follow up with his primary care physician. Though his symptoms resolved without medical therapy, liver enzymes obtained a few weeks later by his primary physician demonstrated progressive elevation and he was referred to the gastroenterology (GI) clinic for further evaluation.

At the time of presentation to the GI clinic, the patient reported a mild sore throat. He denied any recent sick contacts. The past medical history was significant for a Staphylococcus aureus (MRSA) infection 6 months prior for which he was successfully treated with antibiotics. Around the same time, he reports being HIV negative. Since the course of antibiotics, he had not taken any medications, herbal supplements, vitamins, or other over the counter medications, but drank green tea 2-3 times a week. There was no family history of liver disease. The patient was a bisexual male, used alcohol occasionally and denied tobacco use. He had a distant history of inhaled cocaine use, and had never used intravenous drugs nor had he ever received a transfusion.

On physical examination, the patient was a well-developed, well-nourished African American male who appeared comfortable. Vital signs revealed a blood pressure of 137/89 and pulse of 67. There was no scleral icterus, the mouth and oral pharynx appeared normal, and there was no palpable lymphadenopathy. Cardiovascular and pulmonary exams were normal. The abdomen was soft with normal bowel sounds, non-tender, non-distended, and without hepatosplenomegaly, masses, or dullness at the flanks. Both the rectal and genital exams were normal. Skin examination revealed no rashes, jaundice, or stigmata of liver disease.
Liver enzymes were repeated and demonstrated a progressive rise in alkaline phosphatase, aspartate aminotransferase (AST), and alanine aminotransferase (ALT). (Table 1) Additional laboratory studies revealed a complete blood count within normal limits, total bilirubin $1.4 \mathrm{mg} / \mathrm{dL}(0.2-1.2 \mathrm{mg} / \mathrm{dL})$, direct bilirubin 0.5 $\mathrm{mg} / \mathrm{dL}$ (0.0-0.4 mg/dL), PT 13.3 seconds (normal 11.1-15.5 seconds) with INR 1.0 (normal 0.80-1.21), and PTT 56 seconds (normal 19- 39 seconds). The initial work-up for abnormal liver enzymes revealed the following: hepatitis A IgG/IgM antibody, hepatitis B PCR, hepatitis C PCR, CMV quantitative PCR, antinuclear antibody (ANA), antimitochondrial antibody (AMA), anti-liver kidney microsomal (anti-LKM) antibody were all within normal limits, and ceruloplasmin was $109 \mathrm{mg} / \mathrm{dL}$ (normal $22-58 \mathrm{mg} / \mathrm{dL}$ ). An ultrasound of the liver and biliary tree was unremarkable.

A liver biopsy was entertained, but before proceeding an additional test was ordered based on the history of rash and pattern of liver enzyme elevation - a rapid plasma reagin (RPR). The RPR was reactive with a titer of $1: 256$. A confirmatory fluorescent treponemal antibody absorbed (FTA-ABS) was reactive and the patient was diagnosed with secondary syphilis with hepatic involvement. Upon diagnosis, the patient reported being informed of negative test results for both syphilis and HIV approximately 6 months prior to this presentation. He was treated with 2.4 million units of benzathine penicillin $G$ intramuscularly. Given the new diagnosis of a sexually transmitted disease, repeat HIV testing was also recommended and was negative. As presented in Table 1, the liver enzymes improved rapidly following treatment.

\begin{abstract}
Discussion
Syphilis is a complex disease caused by the spirochete Treponema pallidum. More prevalent in the pre-antibiotic era, this infectious disease remains a global health issue today. The disease was the leading cause of neurologic and cardiovascular disease among the middle-aged population at the turn of the 20th century. ${ }^{1}$ Many notable figures have been suspected of having syphilis including Ivan the Terrible, Napoleon Bonaparte and Ludwig van Beethoven. In modern times, the number of cases of
\end{abstract}

Table 1. Liver Enzyme History

\begin{tabular}{|c|c|c|c|c|c|c|}
\hline & $\begin{array}{l}\text { Initial Presentation } \\
\text { (time 0) }\end{array}$ & +5 weeks & +9 weeks & +10 weeks & $\begin{array}{l}\text { Post-Treatment } \\
\text { (2 weeks) }\end{array}$ & $\begin{array}{l}\text { Post-Treatment } \\
\text { (8 weeks) }\end{array}$ \\
\hline $\begin{array}{l}\text { Alkaline Phosphatase } \\
\text { (29-92 IU/L) }\end{array}$ & 448 & 1198 & 1412 & 1538 & 439 & 132 \\
\hline AST (7-42 IU/L) & 272 & 184 & 165 & 306 & 63 & 41 \\
\hline ALT (1-45 IU/L) & 502 & 304 & 242 & 328 & 78 & 45 \\
\hline \multicolumn{5}{|c|}{ Arrow indicates start of treatment } & \multicolumn{2}{|c|}{$\uparrow$} \\
\hline
\end{tabular}


primary and secondary syphilis in the United States decreased during the 1990s and reached its nadir in 2000 since reporting began in 1941. However, recently the reported number of cases of early syphilis (primary and secondary) in the United States has begun to rise as demonstrated by an increase from 5,979 reported cases in 2000 to 11,181 in $2007 .{ }^{2}$ This infection appears to particularly afflict men who have sex with men (MSM), with this group accounting for $65 \%$ of the cases as of $2007 .^{3}$

Untreated, this disease can span decades and cause a wide array of manifestations affecting multiple organ systems, which accounts for it being referred to as the "great imitator." It can present acutely, or chronically with a more indolent course. Syphilis is typically acquired by contact with a skin or mucosal lesion, often sexually, but may also be acquired congenitally. The natural history of syphilis has been well described and progresses through distinct phases. Primary syphilis is characterized by a chancre, a painless indurated lesion occurring at the primary site of inoculation. The time from inoculation to presentation of chancre is inversely proportional to inoculating dose, but typically this incubation period ranges from 10 to 90 days. ${ }^{4}$

About 25 percent of individuals with untreated primary infection will develop secondary syphilis, usually occurring 2 to 8 weeks after appearance of the chancre. ${ }^{5}$ Secondary (disseminated) syphilis most commonly involves the skin as a maculopapular rash involving palms and soles; however, the disease can involve almost any organ in the body. Mucocutaneous, renal, neurologic, gastrointestinal, hepatic and pulmonary manifestations have all been reported. ${ }^{6}$ Constitutional symptoms such as fever, headache, malaise, anorexia, sore throat, myalgias, and weight loss may be present. As the manifestations of secondary syphilis subside, the disease enters a latent period. Latent syphilis is characterized by positive serologies with no clinical signs of infection. Relapse of secondary syphilis can occur as late as 4 years after initial contact, but 75 percent of relapses occur within the first year. Cellular immunity is thought to play a role in the pathogenesis. About one third of untreated patients with latent syphilis develop tertiary syphilis in as early as one year, or as late as 25 to 30 years, after initial infection. ${ }^{5}$ Tertiary syphilis presents with symptomatic involvement of the central nervous system (CNS), cardiovascular system, or the skin and subcutaneous tissues. CNS findings can include meningitis, general paresis, tabes dorsalis (disease of the posterior columns of spinal cord) and meningovascular disease. Cardiovascular disease mainly involves the aortic root, which can lead to aortic insufficiency. Gummatous syphilis can manifest as ulceration on the skin or a mass lesion involving the viscera. ${ }^{5}$

Secondary syphilis, the diagnosis in the patient we have described, is an uncommon and often overlooked cause of hepatitis. ${ }^{7}$ Unfamiliarity with the wide-ranging clinical presentations of secondary syphilis may delay or prevent diagnosis, unfortunate given the availability of highly effective therapy. Approximately $10 \%$ of patients with secondary syphilis may have liver enzyme abnormalities, but clinically apparent hepatitis is rare. ${ }^{8}$ The exact mechanism of disease is unknown but it has been proposed that direct portal venous inoculation and immune complex-mediated processes may be involved. ${ }^{9}$ As in this case, the pattern of liver enzymes found in syphilitic hepatitis often has a cholestatic pattern with a disproportionate elevation of alkaline phosphatase and a less prominent elevation of aminotransferases. One study of HIV-positive patients with syphilitic hepatitis showed alkaline phosphatase levels ranging from 234 to $1870 \mathrm{IU} / \mathrm{L}$ at time of diagnosis. ${ }^{10}$ Hyperbilirubinemia is not commonly seen. ${ }^{11}$ Liver biopsy, generally unnecessary if the diagnosis is entertained and appropriate serologic testing obtained, demonstrates nonspecific findings including periportal lymphocytic infiltration with focal necrosis around central veins, portal areas, and lobules. Trepenomes or granulomatous changes are seen in nearly half of cases. ${ }^{12}$

T. pallidum, the organism responsible for syphilis, cannot be cultured in a laboratory. The quickest and the most direct method of diagnosing primary and secondary syphilis is by direct visualization of exudate from the primary lesion using darkfield microscopy. This requires specialized equipment and experienced technicians to recognize the spirochetes. The use of this method is limited to clinics dedicated to diagnosing and treating sexually transmitted diseases. An alternative method of direct spirochete visualization is the use of direct fluorescent antibody testing (DFA-TP). DFA-TP utilizes flourescence microscopy to examine specimens incubated with fluorescein-labeled anti-T. pallidum globulin. This method also requires specialized equipment and trained personnel which is not widely availability, and therefore most patients suspected of having syphilis are diagnosed using an indirect method serologic testing. ${ }^{13}$ There are two types of serologic tests. The first type is the nontreponemal test, which detect antibodies against cardiolipin-cholesterol-lecithin antigens. Examples are the Venereal Disease Research Laboratory (VDRL) and the Rapid Plasma Reagin (RPR). Positive results are reported as an antibody titer, and these tests are relatively inexpensive and easy to perform. The second type of serologic testing is specific treponemal tests, which detect antibodies against the treponemes and/or its cellular components. Examples of these tests are fluorescent treponemal antibody absorption (FTA-ABS) test, microhemagglutination test (MHA-TP) and Treponema pallidum particle aggluitnation assay (TPPA). These are qualitative tests reported as either reactive or non-reactive. Nontreponemal tests are $78 \%$ to $86 \%$ sensitive in primary syphilis and close to $100 \%$ sensitive in secondary syphilis. ${ }^{14}$ Both nontreponemal and treponemal-specific tests can be falsely positive for many reasons including acute febrile illnesses and immunizations. These test abnormalities typically disappear within 6 months. Chronic illnesses are also implicated as causes of false positive testing including autoimmune disorders like 
systemic lupus erythematosus, intravenous drug use, chronic liver disease and HIV infection. False negative results can also occur, and this may be seen in as many as $20-30 \%$ of patients presenting with a chancre of primary syphilis. ${ }^{15}$

Direct visualization techniques, i.e., darkfield microscopy and DFA-TP, are the only definitive methods of diagnosing early syphilis. Current recommendations state that use of only one serologic test is not adequate for making a diagnosis. ${ }^{16}$ For screening purposes in those who are asymptomatic, a VDRL test should be done and those with positive results should get confirmation using a treponemal test. Nontreponemal antibody titer is an indicator of disease activity and thus can be used to monitor disease progress. A four-fold change in titer is considered clinically significant. ${ }^{16}$ Contrary to this, a person with reactive treponeme-specific tests will have lifelong reactivity even if they are not symptomatic and therefore such testing is not appropriate for monitoring disease. Any person with positive treponemal tests should have a nontreponemal test with titer to assess the status of disease. ${ }^{16}$

The recommended treatment regimen for adults with primary, secondary and early latent syphilis is benzathine penicillin $G$ 2.4 million units IM in a single dose. Late latent syphilis or latent syphilis of unknown duration requires three doses of 2.4 million units IM each at one-week intervals. ${ }^{16}$ As seen in our patient, following treatment the liver enzymes rapidly improve and this response to treatment offers further confirmation of the diagnosis. All patients should be reevaluated 6 and 12 months after treatment. A four-fold reduction in titer of the nontreponemal antibody test (e.g., from 1:16 to 1:4) is considered evidence of an appropriate response. The two nontreponemal tests (VDRL and RPR) cannot be compared directly, so serial monitoring should be performed with the same test. Those without symptomatic improvement or a sustained four-fold reduction in nontreponemal titers should be retreated.

Early detection is imperative in a disease like syphilis where definitive treatment is available and devastating permanent consequences are possible if left untreated. Although a case of fulminant hepatic failure requiring liver transplant secondary to syphilitic hepatitis has been reported, patients should not have any sequelae of chronic liver disease if appropriate treatment is given. ${ }^{12}$ Because of the varied presentation of secondary syphilis, the diagnosis of syphilitic hepatitis should be entertained in anyone presenting with vague constitutional symptoms and acute liver dysfunction with a cholestatic pattern when obstruction, primary disorders such as primary biliary cirrhosis and sclerosing cholangitis, and environmental exposure have been excluded. Clinicians need to have a heightened index of suspicion for this disease in patients at higher risk for syphilis including those with HIV infection and MSM in order to eliminate the need for unnecessary and invasive testing and offer patients rapid resolution of their disease.

\section{References}

1. Tramont EC. The impact of syphilis on humankind. Infect Dis Clin North Am 2004;18:101-10.

2. Centers for Disease Control and Prevention. Sexually transmitted diseases surveillance, 2007. Atlanta, GA: U.S Department of Health and Human Services, 2008(Accessed August 2, 2009 at http://www.cdc.gov/std/stats07/toc.htm.).

3. Centers for Disease Control and Prevention. Sexually transmitted disease surveillance 2007 supplement, syphilis surveillance report. Atlanta, GA: U.S. Department of Health and Human Services, Centers for Disease Control and Prevention, 2009 (Accessed August 2, 2009 at http://www.cdc.gov/std/ Syphilis2007/.).

4. Greenstone CL, Saint S, Moseley RH. A hand-carried diagnosis. N Engl J Med 2007;356:2407-11.

5. Tramont EC. Treponema pallidum (Syphilis). In: Mandell, Bennett, Dolin, ed. Principles and practice of infectious diseases. 6th ed. Orlando, FL: Churchill Livingstone, 2005.

6. McPhee SJ. Secondary Syphilis: Uncommon manifestations a common disease. West J Med 1984;140:35-42.

7. Mandach C, Coca C, Capro-Sampara F, et al. A forgotten aetiology of acute hepatitis in immunocompetent patient: syphilitic infection. J Intern Med 2006;259:214-5.

8. Almoujahed MO, Johnson LB, Khatib R. Syphilitic hepatitis presenting with fever and inguinal lymphadenopathy without rash: a case report. Infectious Diseases in Clinical Practice 2002;11:286-7.

9. Ridruejo E, Mordoh H, F., Avagnina A, Mando OO. Severe cholestatic hepatitis as the first symptom of secondary syphilis. Dig Dis Sci 2004;49:1401-4.

10. Mullick CJ, Liappis AP, Benator DA, Roberts AD, Parenti DM, Simon GL. Syphilitic hepatitis in HIV-infected patients: a report of 7 cases and review of the literature. Clin Infect Dis 2004;39:e100-5.

11. Young MF, Sanowski RG. Syphilitic hepatitis. J Clin Gastroenterol 1992;15:175-6.

12. Lo JO, Harrison RA, Hunter AJ. Syphilitic hepatitis resulting in fulminant hepatic failure requiring liver transplantation. J Infect 2007;54:e115-7.

13. Larsen SA, Steiner BM, Rudolph AH. Laboratory Diagnosis and Interpretation of Tests for Syphilis. Clin Microbiol Rev 1995;8:1-21.

14. Golden MR, Marra CM, Holmes KK. Update on syphilis: resurgence of an old problem. J Am Med Assoc 2003;290:1510-4.

15. Hart G. Syphilis tests in diagnostic and therapeutic decision making. Ann Intern Med 1986;104:368-76.

16. Workowski KA, Berman SM. Sexually transmitted diseases treatment guidelines, 2006. MMWR Morb Mortal Wkly Rep 2006;55:22-30. 